Gazi University
Journal of Science
http://dergipark.gov.tr/gujs

\title{
Temperature Waves Phase Optimal Time Lag in the Refrigerated Warehouse Thermal Insulation
}

\author{
Yurii Anatolievich MIRONCHUK ${ }^{1, *(D)}$, Mykhailo Georgievich KHMELNIUK ${ }^{2}$ (D) \\ ${ }^{I}$ Zhytomyr Military Institute named S. P. Korolyov, 10004, Zhytomyr, Ukraine \\ ${ }^{2}$ Odessa National Academy of Food Technologies, 65039, Odessa, Ukraine
}

\author{
Highlights \\ - Search the optimal time lag values of the temperature waves in the refrigerated warehouse walls \\ - Finite difference method for thermal conductivity equation by nonlinear unsteady boundary conditions \\ - The influence of geographical latitude on the dynamics of heat flow through the walls is considered.
}

\begin{tabular}{|c|}
\hline Article Info \\
\hline $\begin{array}{l}\text { Received: } 03 \text { Feb } 2021 \\
\text { Accepted: } 12 \text { Aug } 2021\end{array}$ \\
\hline Keywords \\
\hline $\begin{array}{l}\text { Thermal inertia } \\
\text { Refrigerated } \\
\text { warehouse } \\
\text { Temperature wave } \\
\text { Heat insulation } \\
\text { Phase optimal delay }\end{array}$ \\
\hline
\end{tabular}

\section{INTRODUCTION}

Daily oscillations in external temperature and heat flux intensity of solar radiation create temperature waves in the outer walls of buildings. The thermal inertia of the wall manifests itself as amplitude damping and as phase lag upon passing temperature wave through the wall. The optimal choice of the buildings walls thermal inertia parameters values during design and construction allows to improve energy saving and the general energy characteristics of residential and public buildings. For existing buildings, the value of the walls thermal inertia is used to estimate the energy performance of buildings. There is an international standard "ISO 13786 Thermal characteristics of building elements - Dynamic thermal characteristics Calculation methods" for engineering calculations of the parameters of thermal inertia of walls of buildings [1].

The thorough explanation of the thermal inertia process in the wall presupposes that the amplitude of the temperature wave is decreased (decrement factor - DF) and the temperature wave phase is delayed in time (time lag -TL) while the temperature wave is passing through the thickness of the walls. The oscillation period of the temperature wave does not alter. 
High DF values help to improve conditions for constant temperature maintaining inside buildings. The need to find solutions to provide the proper level of comfort and to reduce energy consumption for heating and air conditioning in residential and public buildings has stimulated research activity and contributed to the publication of a wide range of scholarly works focused on the issue [2-20].

Utilization of the walls thermal inertia to reduce the impact of daily oscillations of environmental parameters on the stability of microclimate ones inside residential and public buildings is also of great practical interest. The adaptation of effects of the walls thermal inertia offers the opportunity to reduce energy consumption for heating, ventilation and air conditioning for both residential and public buildings [3-5]. Thermal inertia of multilayer walls depends on the order of layers from different materials arrangement. The search for a rational order of such layers' arrangement makes it possible to achieve higher values of thermal inertia at the same consumption of materials. The influence of the design features of multilayer walls on the value of their thermal inertia has been considered in [6-8]. Impact of thermophysical properties of materials on thermal inertia was reviewed in [8-11]. A rational choice of building materials is important to reduce the cost of building walls at a given value of their thermal inertia. The research and studies of thermal inertia are carried out by methods of in situ experiments [9, 12-17] and mathematical simulations $[6,7,18-20]$.

Numerous researches are mainly focused on the study and implementation of thermal inertia DF in residential and public buildings. This study was undertaken because of the lack of published research focused on the problem of determining the rational value of the time lag of the temperature waves phase in the fences (walls, ceiling) of large refrigerated warehouses. Such refrigerators are used for off-season storage of large amounts of food. The chambers of such refrigerators have a total cargo volume from several tons to ten thousand tons or more. Thereby they are the largest consumers of electricity. Optimization of their design and operation can reduce energy consumption and improve the quality and duration of storage products The main objective of the study is to find the optimal values of the thermal inertia parameters for cold chambers thermal insulation in different geographic zones and environmental conditions.

\section{METHODS OF WALL THERMAL INERTIA PARAMETERS CALCULATION}

\subsection{Analytical Solution of the Task of the Walls Thermal Inertia Parameters Calculation}

In [1], to calculate the parameters of the thermal inertia of the walls, a method was used based on solving the equation of thermal conductivity of a multilayer plate with a periodically varying surface temperature under boundary conditions of the 1st kind [21]. In $[1,21]$ calculations are performed in matrix form, which is inconvenient for visual analysis of formulas.

The complete analytical solution of the task of walls thermal inertia parameters calculation is given in [22]. This solution has been developed for multi-layer walls for the case of harmonic oscillations of the external temperature at a constant indoor temperature under boundary conditions of the 3-rd kind on the outer surfaces of the walls. The solution [22] allows calculating DF and TL of the temperature wave when it passes through a multi-layer wall provided that thicknesses and materials properties of its layers are known. The solution is shortly described below, following [22].

With harmonic oscillations in the ambient temperature, the temperature of the outer surface of the fence (wall) also has harmonic oscillations

$$
t_{\text {out }}=t_{\text {out } .0}+A_{\text {t.out }} \cdot \operatorname{Cos} \frac{2 \pi}{T}\left(\tau \pm \varphi_{\text {out }}\right)
$$

where $t_{\text {out } .0}$ - average daily temperature of the outer surface of the wall, ${ }^{\circ} \mathrm{C}$;

$A_{\text {t.out }}$ - daily amplitude of the wall outer surface temperature, ${ }^{\circ} \mathrm{C}$;

$T$ - oscillations period;

$\tau$ - time; 
$\varphi_{\text {out }}$ - phase of temperature oscillations of the wall outer surface (measured in units of time).

By passing the temperature wave through the wall (Figure 1), its amplitude decreases, the phase lags, and the oscillations period remains. Thus, the temperature of the inner surface of the wall is

$t_{\text {ins }}=t_{\text {ins. } 0}+A_{t . i n s} \cdot \operatorname{Cos} \frac{2 \pi}{T}\left(\tau \pm \varphi_{\text {ins }}\right)$.

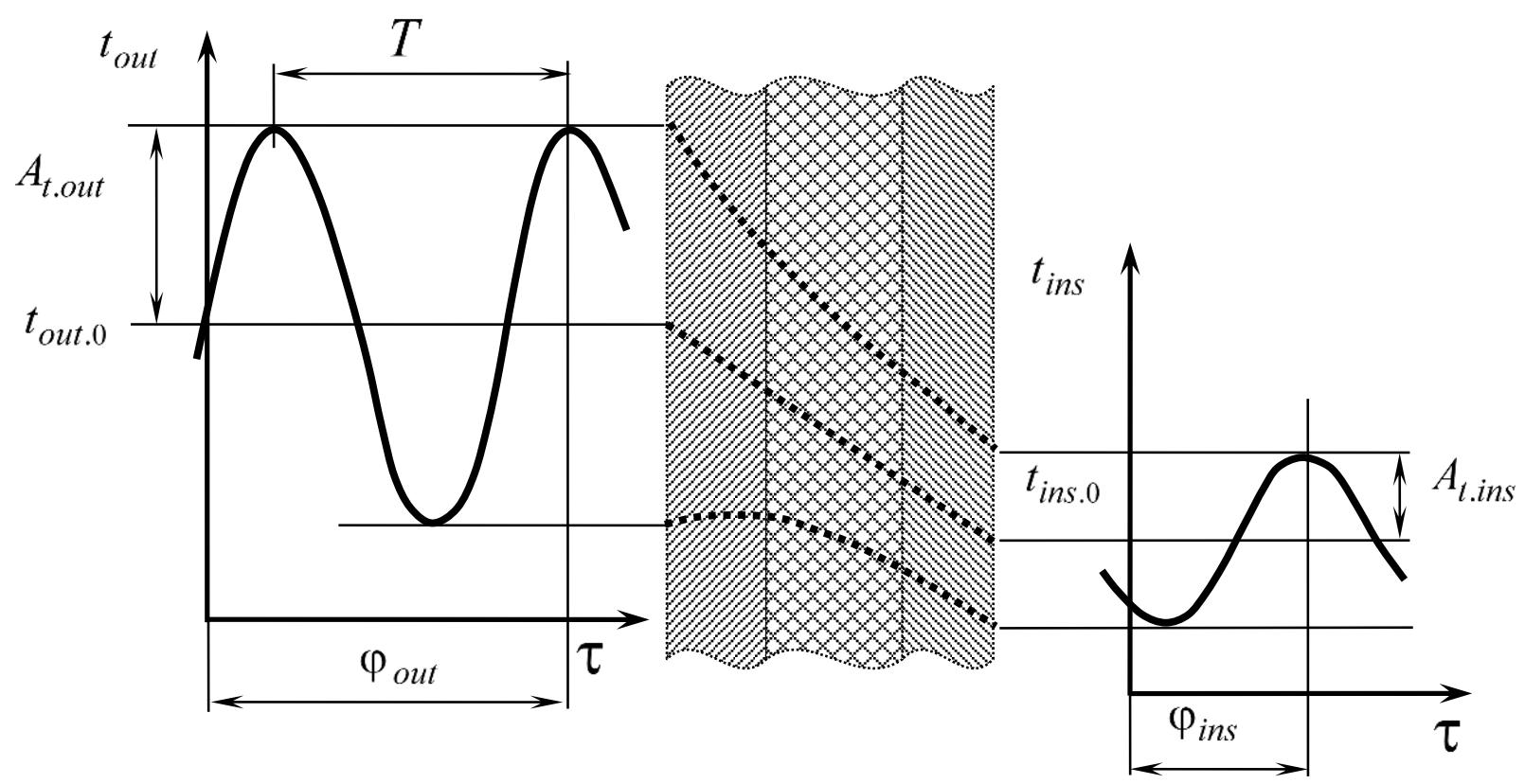

Figure 1. Time Lag and Decrement Factor for temperature wave passing through a multi-layer wall

Temperature wave amplitude damping (DF)

$D F=\frac{A_{\text {t.out }}}{A_{\text {t.ins }}}$.

Temperature wave phase lag (TL)

$T L=\varphi_{\text {out }}-\varphi_{\text {ins }}$.

For the case of harmonic temperature oscillations $(1,2)$, to the differential equation of heat conduction through a single-layer wall corresponds the specific solution, in which the temperature $\dot{t}$ is a complex number, expressed in terms of the hyperbolic sine and the hyperbolic cosine functions

$\dot{t}=e^{\left(\frac{2 \pi \tau}{T} i\right)} \cdot\left[A \cdot \operatorname{ch}\left(x \cdot \sqrt{\frac{2 \pi i}{T a}}\right)+B \cdot \operatorname{sh}\left(x \cdot \sqrt{\frac{2 \pi i}{T a}}\right)\right]$.

Here, $A$ and $B-$ are constants, derived from the boundary conditions;

$a$ - thermal diffusivity of the layer material, $\mathrm{m}^{2} / \mathrm{s}$;

$x$ - coordinate through the wall thickness. 
In (5), the dot above the temperature designation means that the temperature is considered as a radius vector on the complex plane, which rotates at a constant angular velocity with a period $T$.

Equation (5) allows to calculate the value of the temperature $\dot{t}$ radius vector on the complex plane for the randomly assigned time $\tau$, at the randomly assigned distance $x$ from zero coordinates. The DF and TL of the temperature wave in the layer can be found by dividing the complex Equation (5) for the temperature on the outer surface of the layer by the same equation for the temperature on the inner surface of the layer. If this operation is repeated stepwise for every next layer, it is possible to find DF and TL of the temperature wave when it passes through the multilayer wall

$\dot{\beta}=e^{\Sigma(R S \sqrt{i})} \times \frac{S_{1} \sqrt{i}+\alpha_{i n s}}{S_{1} \sqrt{i}+\dot{Y}_{1}} \times \frac{S_{2} \sqrt{i}+\dot{Y}_{1}}{S_{2} \sqrt{i}+\dot{Y}_{2}} \times \ldots \times \frac{S_{n} \sqrt{i}+\dot{Y}_{n-1}}{S_{n} \sqrt{i}+\dot{Y}_{n}} \times \frac{\dot{Y}_{n}+\alpha_{\text {out }}}{\alpha_{\text {out }}}$

In Equation (6) the layers are numbered from the side of the cold store in contrary direction to the direction of heat leakage though the wall. $\dot{\beta}$ is a complex number. Its modulus expresses DF, and its argument expresses the TL of the temperature wave from the environment to the inner wall surface. $\alpha_{\text {ins }}$ and $\alpha_{\text {out }}$ are the coefficients of convective heat transfer for inside and outside surfaces of wall. $R$ is thermal resistance of the material layer. $S$ is the coefficient of the heat assimilation of the layer material. It depends on the oscillation period of the temperature wave and on the heat capacity $C$, density $\rho$, thermal conductivity $\lambda$ of the layer material

$$
S=\sqrt{\frac{2 \cdot \pi \cdot \lambda \cdot C \cdot \rho}{T}}
$$

In Equation (6), $\dot{Y}$ is the heat absorption coefficient of cross - section for any plane section along the wall thickness. $\dot{Y}$ is a complex number. Its modulus is equal to the ratio the amplitude of heat gains oscillations to the amplitude of temperature oscillations in the selected plane section of the wall. The argument characterizes the magnitude of the phases shift between temperature oscillations and heat gain oscillations for the selected plane section.

$$
\dot{Y}_{n}=S \sqrt{i} \frac{\operatorname{th}\left(R_{n} S_{n} \sqrt{i}\right)+\frac{\dot{Y}_{n-1}}{S \sqrt{i}}}{1+\frac{\dot{Y}_{n-1}}{S \sqrt{i}} \cdot \operatorname{th}\left(R_{n} S_{n} \sqrt{i}\right)} .
$$

On the inside surface of the wall $\dot{Y}_{0}=\alpha_{i n s}$.

In Equations (6), (8) the product $R S$ of the layer thermal resistance and the heat absorption coefficient of layer material is called the thermal inertia of the layer

$D_{n}=R_{n} \cdot S_{n}$.

Thermal inertia of multilayer wall is equal to the sum of layers' thermal inertia

$D_{w}=\sum D_{n}=\sum_{i=1}^{n}\left(R_{i} \cdot S_{i}\right)$.

From the analysis of Equations (6) and (8), it can be seen that thermal inertia is the main factor, on which the values of TL and DF depend. For a multi-layer wall, TL and DF additionally depend on the order of 
layers in the wall, on the intensity of heat transfer on the wall surfaces, on the presence and thickness of air interlayers inside the wall.

The analytical solution [20] reveals the physical nature of the TL and DF, shows the factors on which their intensity depends. But the direct use of [22] for engineering calculations is not accessible, since the conditions, for which the analytical solution was obtained, differ from the actual operating conditions of the fences of cold stores. First, the daily fluctuations in external temperature are far from harmonic. Second, the real nature of the heat gain from solar radiation does not correspond to the boundary conditions adopted in [22].

Third, the real boundary conditions are essentially nonlinear. Also, the reason for nonlinearity may be the dependence of the thermophysical properties of the layers materials on temperature. The combination of these factors determines the need to apply numerical methods for the engineering analysis of this problem.

\subsection{The Real Scheme of Heat Transfer Through the Walls of the Cold Store}

During the daytime, the outer surface of the wall absorbs the energy of solar radiation $q_{\text {san }}$ and heats up to a temperature higher than the outside temperature. One part of heat from the heated outside surface of wall is withdrawn by convection to colder atmospheric air $-q_{\text {conv }}$. The second part of heat is removed by infrared radiation into the environment $-q_{\text {rad }}$. Remaining portion of heat is gone into the cold store by thermal conductivity through the wall $-q_{\lambda}$. As an illustration, Figure 2 shows a diagram of heat transfer through a flat roof of the cold store.

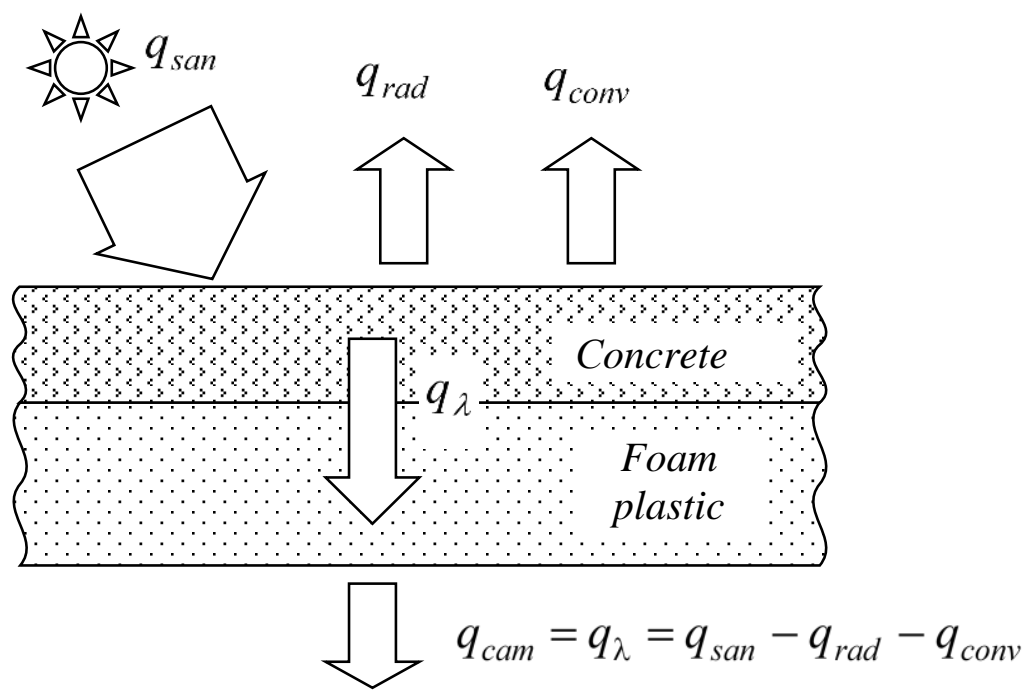

Figure 2. Heat transfer through the flat roof of the cold room

The boundary conditions are substantially non-linear as the $q_{\text {san }}$ intensity varies throughout the day. As a consequence, the temperature of the outer surface of the wall and the difference between the surface temperature and the ambient temperature change. This is accompanied by a change in the intensity of the $q_{\text {rad }}$ and $q_{\text {conv }}$. The dependence of the thermophysical properties of wall materials on temperature creates an internal nonlinearity.

\subsection{Numerical Solution of the Thermal Conductivity Equation}

The various variants of the finite difference method are the most common for the thermal conductivity equation numerical solution [23]. Explicit method, implicit method, Crank-Nicholson method are 
convenient to solve linear equations. The implicit method is very popular because it is absolutely stable and it allows to integrate linear equations with a large time step.

For equations with high nonlinearity the numerical integration with small time steps is required for getting possibility to make corrections on nonlinearity at each time step [24]. Under such conditions, it is easier to use an explicit scheme with a simple computational algorithm. The explicit scheme is stable only at small time steps of integration, not exceed the critical value

$$
\Delta \tau \leq \frac{C \cdot \rho \cdot \delta^{2}}{2 \lambda} .
$$

In (11), $\delta$ is the step of spatial grid.

For the numerical integration of equations with high nonlinearity, it is more convenient to use the method of elementary heat balances. This method was proposed in [25] for linear equations and improved in [26] for nonlinear equations.

When creating a numerical model, the method with iterative calculation of the surface temperature was applied to approximate complex nonlinear boundary conditions at each time step [27]. Such approximation of the boundary conditions provides high convergence and stability of the computational algorithm, which makes it possible to perform calculations with high accuracy at a small number of nodes for the spatial grid. From (11) it is easy to observe that if the step of the spatial grid is doubled, the number of nodes will be halved and the time step for one node will be increased by 4 times. In total, it will reduce the calculations duration for the wall by 8 times.

The created numerical model was programmed in Pascal. The approximation property of the mathematical model was verified by comparing the simulation results with analytical calculations according to [22].

For the necessary simulation of the refrigerated warehouse operation, the developed program also includes a refrigeration machine model and an environment model.

\subsection{Simulation of Refrigeration Machine}

The simplest model of an ammonia refrigeration machine was used. Its single-stage cycle is shown in Figure 3 . The calculation of the cycle parameters was carried out following the recommendations [28, 29].

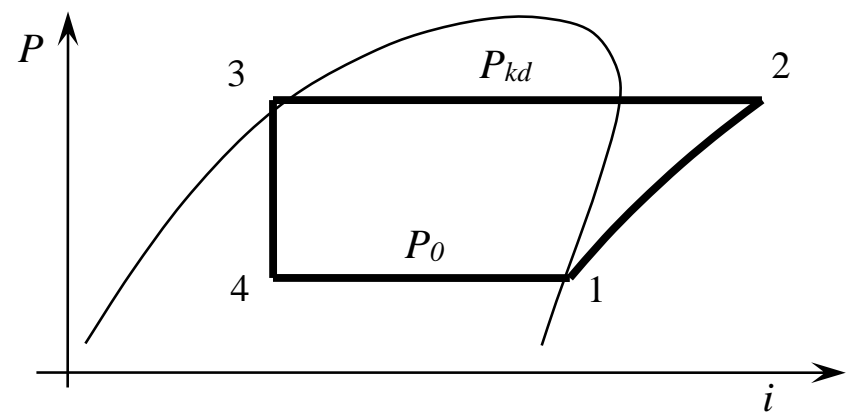

Figure 3. Model operation cycle of single stage ammonia refrigeration machine

The refrigeration cycle parameters are determined by evaporating temperature and condensing one. Evaporating temperature is set depending on the required temperature in the cold store and then it is kept constant. The condensing temperature and pressure are automatically self-adjusting depending on the ambient temperature. Since the temperature of the outside air is continuously changing, the temperature and pressure of condensation also change. At the same time key parameters of the refrigeration machine 
performance as coefficient of performance, volumetric capacity coefficient, and compressor efficiency also continuously change in accordance with condensation temperature.

The refrigeration coefficient of performance (performance energy ratio) characterizes how much artificial cold is produced per unit of consumed power. For the ideal Carnot refrigeration cycle we have

$\varepsilon=\frac{T_{0}}{T_{k d}-T_{0}}$.

Volumetric productivity of piston compressor equals

$\lambda=\frac{T_{0}}{T_{k d}} \cdot\left(1-c \cdot\left[\left(\frac{P_{k d}}{P_{0}}\right)^{\frac{1}{m}}-1\right]\right)$.

Adiabatic efficiency of ammonia piston compressor equals

$\eta_{i}=\frac{T_{0}}{T_{k d}}+0,001 \cdot\left(T_{0}-273\right)$.

The mechanical power of piston compressor which is required to produce refrigerating capacity $Q_{\text {wall }}$

$N_{K M}=\frac{1}{\eta_{i}} \cdot \frac{Q_{\text {wall }}}{\left(i_{1}-i_{4}\right)} \cdot \frac{k}{k-1} \cdot P_{1} \cdot v_{1} \cdot\left[\left(\frac{P_{k d}}{P_{1}}\right)^{\frac{k-1}{k}}-1\right]$.

According to Equations (12) - (15), with an increase in the condensing temperature and pressure, volumetric productivity and adiabatic efficiency of the compressor decrease, and the specific work of the refrigerant compression increases. Consequently, in the daytime, specific energy consumption and volumetric capacity of compressor, required to produce a unit of refrigeration will be higher than at night. In this regard, the issue of TL utilization so that the daytime peak of heat flow to the outer surfaces of the walls can come to the cold store at night is still relevant.

\subsection{Real Character of Environmental Parameters Daily Oscillations}

Daily oscillations of the external temperature are not harmonic. The minimum air temperature is observed before sunrise. The maximum air temperature is observed in 2-3 hours after noon local solar time. The graph of the daily oscillations of the external temperature is no symmetric, as shown in Figure 4. The degree of asymmetry depends on both the geographical latitude of the area and the season of the year.

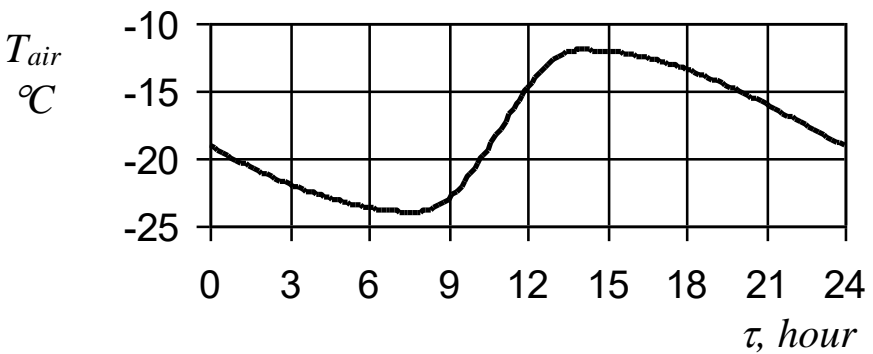

Figure 4. The typical daily variation of air temperature in the middle latitudes of the continent for winter time 
The intensity of the heat input by solar radiation depends on the daytime, season, latitude of the area, orientation of the irradiated surface, transparency of the atmosphere, the presence of clouds, the presence of precipitation, the presence or absence the shade from trees or neighbouring buildings.

\section{THE RESEARCH FINDINGS AND DISCUSSION}

Simulation of nonlinear nonstationary processes was performed for a cold store with a flat roof, the temperature of frozen foodstuffs was $-20^{\circ} \mathrm{C}$. The thermal resistance was set at $5 \mathrm{~m}^{2} \cdot \mathrm{K} / \mathrm{W}$. The outer surface is considered as a grey body with a blackness level of 0.9 , which corresponds to a flat roof contaminated with sand. The estimated season is July 1 , which corresponds to the annual maximum ambient temperature after the summer solstice in the northern hemisphere.

Comparative calculations were performed for two variants of the flat roof. The wall made of sandwich panels (the sheets of foam plastic covered with a protective thin-walled metal shell) could be referred to as an example of the wall with low thermal inertia. Thickness of the metal shell is minor and therefore its contribution to the thermal resistance and thermal inertia of the sandwich panel is negligible and can be ignored. Taking into consideration all of the above, the wall with low thermal inertia was simulated as one foam plastic layer without a protective shell.

To simplify the model, walls with high thermal inertia were considered as consisting of only two layers of material. The concrete layer provided the required wall strength. The foam layer provided thermal insulation. Thermal resistance of two-layer walls equals

$R=R_{C}+R_{F . P .}=\frac{\delta_{C}}{\lambda_{C}}+\frac{\delta_{F . P .}}{\lambda_{F . P .}}$

Thermal inertia of two-layer wall

$D=R_{C} \cdot S_{C}+R_{F . P .} \cdot S_{F . P .}=\frac{\delta_{C}}{\lambda_{C}} \cdot S_{C}+\frac{\delta_{F . P .}}{\lambda_{F . P .}} \cdot S_{F . P .}$

Since $\lambda_{C}>>\lambda_{F . P .}$ and $S_{C} \gg S_{F . P .}$, on the basis of Equations (16), (17) at a specified value $R$ for wall, it is possible to obtain the required value $D$ of the wall by choosing the ratio between the layer thickness of concrete $\delta_{C}$ and the layer thickness of foam plastic $\delta_{F . P .}$. When performing the calculations, it was selected the value of the thermal inertia of a two-layer wall, at which TL provided a shift of the night time minimum heat leakage through the wall for the time of the daytime maximum of the external temperature.

\subsection{Time-Lag Impact on Refrigeration System Technical Characteristics}

The influence of TL in the flat roof on the daily dynamics of the operating mode of the refrigeration system is presented in Figure 5. In Figure 5 all values are given relative to $1 \mathrm{~m}^{2}$ of roof surface. Geographic location and climatic conditions are given for Odessa city, Ukraine. 


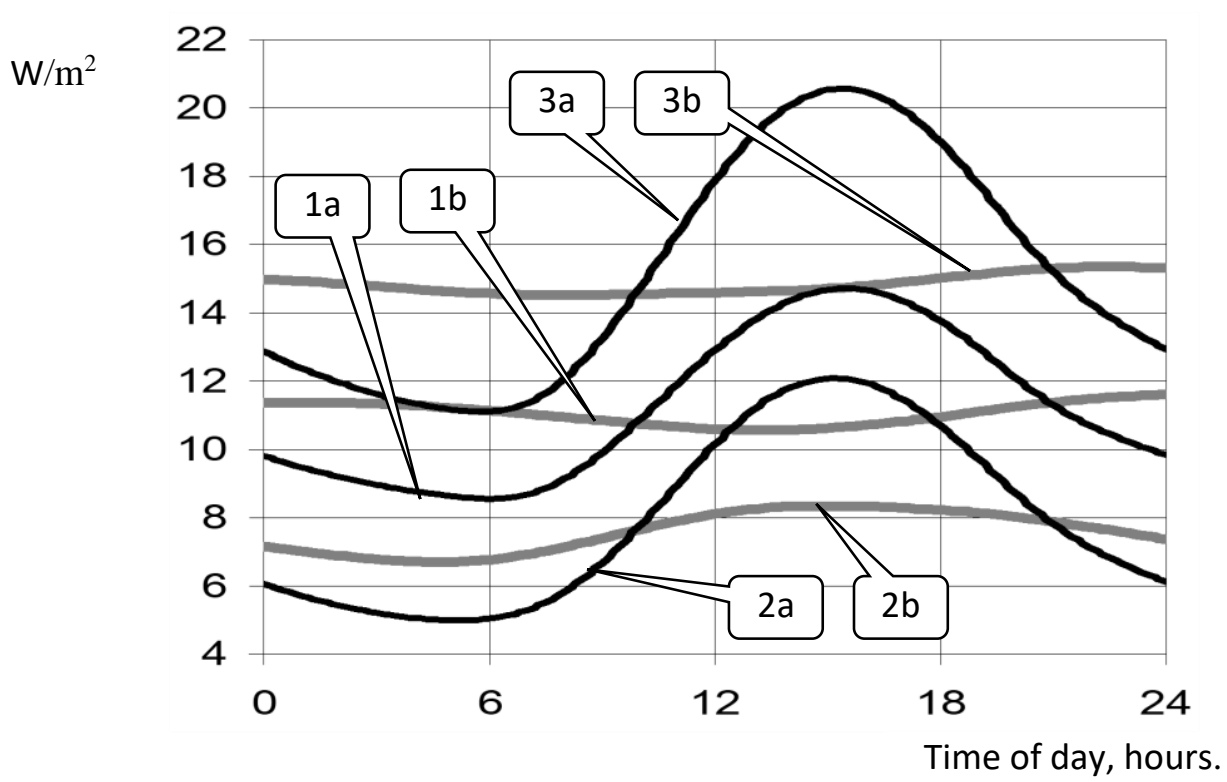

Figure 5. TL impact in a flat roof on the daily dynamics of the operating mode of the refrigeration system: 1 - heat leakage into camera through the flat roof; 2 - effective power of compressor;

3 - thermal load of the condenser. a - low thermal inertia of the wall (black lines); $b$ - high thermal inertia of the wall (grey lines)

In case when the thermal inertia of the wall is equal to zero, the time lag is absent and the current value of the heat gain into the cold store at the current time $\tau$ depends on the temperature of environment $t_{\text {own }}$ at the same current time $\tau$

$q_{\text {cam }}(\tau)=\frac{1}{R} \cdot\left[t_{\text {own }}(\tau)-t_{\text {cam }}\right]$.

At zero thermal inertia, the maximum heat leakage through the wall enters the chamber during the hottest time of the day. In this case, the refrigeration machine must operate with the maximum condensing temperature and the minimum values of the coefficient of performance (12), volumetric productivity (13) and adiabatic efficiency (14) of the compressor. At this time the maximum volumetric productivity of the compressor and the maximum mechanical power (15) are required to produce cold.

If the thermal inertia of the wall does not equal to zero, TL takes place in the wall. In this case the current value of the heat gain input into the cold store at the time $\tau$ is determined by the temperature of environment at the time $(\tau-T L)$

$q_{\text {cam }}(\tau) \approx \frac{1}{R} \cdot\left[t_{\text {own }}(\tau-T L)-t_{\text {cam }}\right]$.

Equation (19) is illustrative and approximate because it does not take into consideration the effect of DF in the wall, as can be seen in Figure 5. As follows from (6) the values DF and TL are interrelated. This interrelation depends of layers' number, their order, thickness and thermophysical properties of layers' materials.

With a correctly assigned thermal inertia, the TL value is such that the minimum daily value of the heat leakage through the wall is shifted by the time of the maximum daily ambient temperature. If the TL value is high, the DF value will also be high. Due to this, the amplitudes of daily fluctuations of the heat leakage through the wall and the amplitudes of the effective power of the compressor and the amplitude of the heat load of the condenser are reduced. Taken together, this makes it possible to reduce the installed volumetric 
productivity of the compressor, the heat exchange area of the condenser and chamber evaporators, the volumes of the receivers and the diameters of the pipelines.

\subsection{Climatic Conditions Impact}

The expected efficiency of TL application depends on the climatic conditions of the cold store location. The main parameters that determine the daily dynamics of the environment state are the daily amplitude of temperature fluctuations of the atmospheric air and amplitude of the heat gain intensity oscillations from solar radiation. The air temperature daily amplitude depends on the climatic conditions of the area - for example, over the surface of the oceans it is $2 \div 4^{\circ} \mathrm{C}$, and in deserts it can be more than $30^{\circ} \mathrm{C}$. The intensity of heat gain by solar radiation depends on the daytime and season, the geographical latitude of the area, the angle of inclination of the surface to the vertical, the angle of inclination of the surface to the geographic meridian.

In the process of simulation for the calculation of heat gains from solar radiation, the geographical latitude is set to $40^{\circ}$. In the North Hemisphere at this latitude there are densely populated industrialized territories and the capitals of many countries (USA, Portugal, Spain, Italy, Greece, Turkey, Transcaucasia, Central Asia, China, Japan) with different types of climate - from oceanic to sharply continental. Since for all the indicated territories there are different values of the maximum and average annual air temperature, when carrying out calculations to achieve the conditions of comparability, it is assumed that the average daily temperature for the calculation period is $25^{\circ} \mathrm{C}$.

Daily amplitude impact of the air temperature on the daily amplitude of the heat leakage through the wall is presented in Figure 6. In this figure the lines of the same color show the maximum and minimum daily values of heat gain. The distance between the lines of the same color represents the daily amplitude of the heat gain. At zero amplitude of the air temperature, the amplitude of heat gain was determined by the intensity of solar radiation.

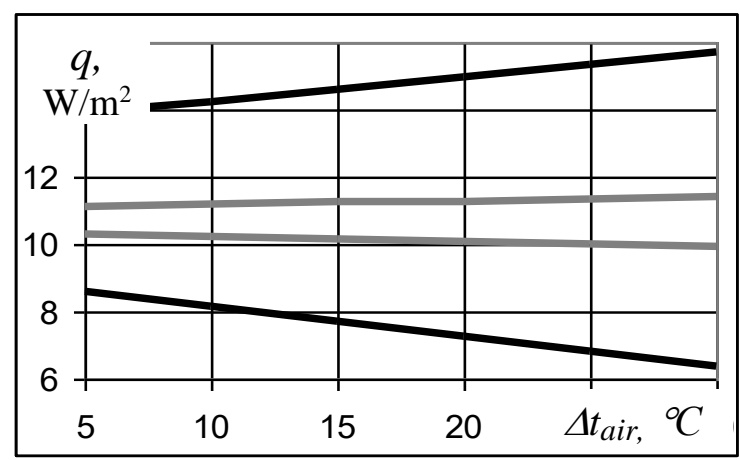

Figure 6. Daily amplitude dependence of the heat leakage into the cold store through a flat roof on the daily amplitude of the air temperature with lowly inertial (black lines) and highly inertial (grey lines) thermal insulation

The influence of the daily amplitude of the atmospheric air temperature (in the presence of a heat gain from solar radiation) on the daily dynamics of the operating mode of the refrigeration unit is shown in Figure 7. 


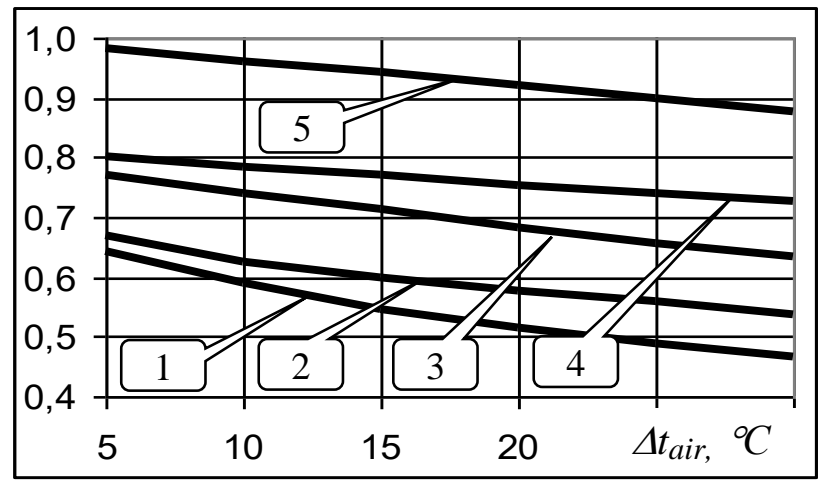

Figure 7. Relative decrease depending on the ambient temperature amplitude: 1 - maximum volumetric productivity of compressor; 2 - maximum effective power of compressor; 3 - maximum heat power of condenser; 4 - maximum specific heat leakage into the camera through the wall; 5 - daily consumption of electrical power

From the analysis of Figures 6 and 7 it can be seen that the use of TL is more effective for climatic zones with high daily amplitude of atmospheric air temperature at a high intensity of the solar radiation gain. Since TL shifts the heat gain from solar radiation into the chamber from day to night, a positive effect is achieved even at zero daily amplitude of the ambient air temperature.

\section{CONCLUSION}

In the design of refrigerators, it is necessary to ensure the optimal TL in the insulated walls and roof. At the same time, high DF values will also be provided. This will reduce the peak heat leakage through the walls to the average daily level. As a result, a lower installed compressor displacement and a lower installed compressor motor drive power will be required. The design values of the heat exchange areas of the condenser and chamber evaporators, as well as the volumes of receivers and the diameters of pipelines, will decrease.

Improving the conditions for maintaining a stable temperature in the cold stores contributes to increase in the quality and increase in the shelf life of food products in the refrigerator.

With favorable ratios between the daily amplitude and the average daily temperature of the ambient air and the temperature in the cold stores, the optimization of TL can slightly reduce the total amount of electricity consumed to remove heat leakage through the walls.

Optimization of TL for wall thermal insulation reduces the value of peak electrical power consumption of refrigerated warehouse to the average daily level. This open the way for reducing the installed capacity of electrical equipment, for reducing the cross-sections of power cables, for reducing peak loads on power lines. It also helps to reduce the unevenness of the daily load graphs at the power plants.

\section{CONFLICTS OF INTEREST}

No conflict of interest was declared by the authors.

\section{REFERENCES}

[1] ISO 13786 Thermal performance of building components - Dynamic thermal characteristics Calculation methods, European Committee for Standardization, 22, (2007).

[2] Verbeke, S., Audenaert, A., "Thermal inertia in buildings: a review of impacts across climate and building use", Renewable and Sustainable Energy Reviews, 82(3): 2300-2318, (2018). 
[3] Aste, N., Angelotti, A., Buzzetti, M., "The influence of the external walls thermal inertia on the energy performance of well insulated buildings", Energy and Buildings, 41(11): 1181-1187, (2009).

[4] Hu, R., Liu, G., Niu, J., "The impacts of a building's thermal mass on the cooling load of a radiant system under various typical climates", Energies, 13(6): 1356, (2020).

[5] Williams, S., Short, M., Crosbie, T., "On the use of thermal inertia in building stock to leverage decentralised demand side frequency regulation services", Applied Thermal Engineering, 133: 97106, (2018).

[6] Asan, H., "Investigation of wall's optimum insulation position from maximum time lag and minimum decrement factor point of view", Energy and Buildings, 32(2): 197-203, (2000).

[7] Bellahcene, L., Cheknane, F., Bekkouche, S., Sahel, D., "The effect of the thermal inertia on the thermal transfer in building wall”, E3S Web Conf., 22: 00013, (2017).

[8] Oktay, H., Argunhan, Z., Yumrutaş, R., Işı1k, M., Budak, N., "An investıgation of the influence of thermophysical properties of multilayer walls and roofs on the dynamic thermal characteristics", Mugla Journal of Science and Technology, 2(1): 48-54, (2016).

[9] Asan, H., Sancaktar, Y. S., "Effects of Wall's thermophysical properties on time lag and decrement factor", Energy Build, 28(2): 159-166, (1998).

[10] Avendaño-Vera, C., Martinez-Soto, A., Marincioni, V., "Determination of optimal thermal inertia of building materials for housing in different Chilean climate zones", Renewable and Sustainable Energy Reviews, 131: 110031, (2020).

[11] Oktay, H., Yumrutaş, R., Argunhan, Z., "An experimental investigation of the effect of thermophysical properties on time lag and decrement factor for building elements", Gazi University Journal of Science, 33(2): 492-508, (2020).

[12] Evangelisti, L., Guattari, C., Gori, P., Asdrubali, F., "Assessment of equivalent thermal properties of multilayer building walls coupling simulations and experimental measurements", Building and Environment, 127: 77-85, (2018).

[13] Neya, I., Yamegueu, D., Coulibaly, Y., Messan, A., Sountong-Noma Ouedraogo, A., "Impact of insulation and wall thickness in compressed earth buildings in hot and dry tropical regions", Journal of Building Engineering, 33: 101612, (2021).

[14] Orosa, J.A., Oliveira, A. C., "A field study on building inertia and its effects on indoor thermal environment", Renewable Energy, 37(1): 89-96, (2012).

[15] Rakshit, D., O'Leary, T., Hogan, R., Robinson, A., Byrne, A., "The use of three-dimensional conjugate CFD to enhance understanding of, and to verify, multi-modal heat transfer in dynamic laboratory test walls", Civil Engineering Research in Ireland, 3: 364-369, (2020).

[16] Toure, P., Dieye, Y., Momar Gueye, P., Sambou, V., Bodian, S., Tiguampo, S., "Experimental determination of time lag and decrement factor", Case Studies in Construction Materials, 11: e00298, (2019).

[17] Ulgen, K., "Experimental and theoretical investigation of effects of wall's thermophysical properties on time lag and decrement factor", Energy and Buildings, 34(3): 273-278, (2002).

[18] Asan, H., "Numerical computation of time lags and decrement factors for different building materials", Building and Environment, 41: 615-620, (2006). 
[19] Kontoleon, K., Eumorfopoulou, E., "The influence of wall orientation and exterior surface solar absorptivity on time lag and decrement factor in the Greek region", Renewable Energy, 33(7): 16521664, (2008).

[20] Netam, N., Sanyal, S., Bhowmick, S., "A mathematical model featuring time lag and decrement factor to assess indoor thermal conditions in low-income-group house", Journal of Thermal Engineering, 6(2): 114-127, (2020).

[21] Carslaw, H., Jaeger, J., Conduction of Heat in Solids, 2nd edition. Oxford University Press, London, (1959).

[22] Shklover, A. M., Heat transfer under periodic thermal influences, Gosenergoizdat, Moskow, 160, (1961).

[23] Samarskii, A., Goolin, A., Numerical methods, Moskow, Nauka, Main Editorial bord for Physical and Mathematical Literature, 432, (1989).

[24] Belyaev, N., Ryadno, A., Mathematical methods of heat conduction: textbook, Kiev, Higher school, 415, (1992).

[25] Vanichev, A. P., "An approximate method for solving heat conduction problems with variable constants", Proceedings of the Academy of Sciences of the USSR, Department of Engineering Sciences, 12: 1767-1774, (1946).

[26] Voitko, A. A., "Heat and mass transfer when freezing fruits and vegetables in fluidization apparatuses", $\mathrm{PhD}$ Thesis, Odessa Institute of Low-Temperature Engineering and Energetics, Odessa, 310, (1989).

[27] Mironchuk, Yu. A., "Approximation of compicated nonlinear boundary conditions in finitedifference modeling of heat exchange processes in cold chambers thermal insulation", Refrigeration Engineering and Technology, 4(78): 17-20, (2002).

[28] Chumak, I., Nikulshina, D., Refrigeration units. Design: Textbook manual for universities, Kiev: Higher school, Head Publishing House, 280, (1988).

[29] Chumak, I., Lagutin, A., Chepurnenko, V., Laryanovsky, S., etc. Refrigeration units. Design: Textbook, Edited by Doctor of Technical Sciences Professor Chumak I., - 3rd editions, revised and enlarged, Odessa: Druk, 480, (2007). 